\title{
A note on radar altimeter signatures of Internal Solitary Waves in the ocean
}

\author{
J.C.B. da Silva ${ }^{a}{ }^{*}$ \& A.L.F. Cerqueira ${ }^{a}$ \\ ${ }^{a}$ Faculdade de Ciências da Universidade do Porto - Departamento de Geociências, Ambiente e Ordenamento \\ do Território - Rua do Campo Alegre, 687, 4169-007 Porto, Portugal \\ ${ }^{\mathrm{b}}$ CIMAR/CIIMAR - Interdisciplinary Centre of Marine and Environmental Research, University of Porto, \\ Rua dos Bragas 289, 4050-123 Porto, Portugal
}

\begin{abstract}
It is well known that Internal Waves of tidal frequency (i.e. Internal Tides) are successfully detected in seasurface height (SSH) by satellite altimetry ${ }^{[1]}$. Shorter period Internal Solitary Waves (ISWs), whose periods are an order of magnitude smaller than tidal internal waves, are however generally assumed too small to be detected with standard altimeters (at low sampling rates, i.e. $1 \mathrm{~Hz}$ ). This is because the Radar Altimeter (RA) footprint is somewhat larger, or of similar size at best, than the ISWs typical wavelengths. Here it will be demonstrated that new generation high sampling rate satellite altimetry data (i.e. $\sim 20 \mathrm{~Hz}$ ) hold a variety of short-period signatures that are consistent with surface manifestations of ISWs in the ocean. Our observational method is based on satellite synergy with imaging sensors such as Synthetic Aperture Radar (SAR) and other high-resolution optical sensors (e.g. 250m resolution MODIS images) with which ISWs are unambiguously recognized. A first order commonly accepted ISW radar imaging mechanism is based on hydrodynamic modulation models ${ }^{[2]}{ }^{[3]}$ in which the straining of surface waves due to ISW orbital currents is known to cause modulation of decimeter-scale surface waves, which have group velocities close to the IW phase velocity. This effect can be readily demonstrated by measurements of wind wave slope variances associated with short-period ISWs, as accomplished in the pioneer work of Hughes and Grant ${ }^{[4]}$. Mean square slope can be estimated from nadir looking RAs using a geometric optics (specular) scattering model ${ }^{[5][6][7]}$, and directly obtained from normalized backscatter (sigma0) along-track records. We use differential scattering from the dual-band (Ku- and C-bands) microwave pulses of the Jason2 high-rate RA to isolate the contribution of small-scale surface waves to mean square slope. The differenced altimeter mean square slope estimate, derived for the nominal wave number range 40-100 rad/m, is then used to detect ISWs in records of along-track high sampling rate RAs. The RA signatures of these ISWs are also apparent in radar backscattered pulse waveforms from the original Sensor Geophysical Data Records (SGDR), in high resolution (20-Hz) data. The shape of these waveforms is modified by the ISWs with respect to waveforms unperturbed by short-period internal waves. Hence, a new method for identification of ISWs in high-rate RA records that combines along-track differenced mean square slopes across ISW crests and waveform shape variation is put forward in this paper. Validation of the method is warranted with quasi-coincident (in time and space) SAR images of ISWs in various deep ocean regions, such as the Andaman Sea, the Mascarene Ridge of the Indian Ocean and the North Atlantic tropical ocean. The practical significance of this new method is related to the anticipated SWOT wide-swath altimeter mission as well as the recently launched Sentinel-3A SARAL, for which removal of internal wave signals may be of critical importance for observing other high-frequency sub-mesoscale dynamics.
\end{abstract}

Keywords: Internal Solitary Waves, Radar Altimeter, Sigma0 blooms

*jdasilva@fc.up.pt; phone: +351 220402476; fax: +351 220402490

Remote Sensing of the Ocean, Sea Ice, Coastal Waters, and Large Water Regions 2016, edited by Charles R. Bostater, Jr., Xavier Neyt, Caroline Nichol, Oscar Aldred, Proc. of SPIE Vol. 9999, $999902 \cdot$ ( ) 2016 SPIE · CCC code: 0277-786X/16/\$18 · doi: 10.1117/12.2240870 


\section{INTRODUCTION}

\subsection{Altimeter backscatter and sea surface roughness}

The basic concept of satellite altimetry is simple. The main objective is to measure the range $\mathrm{R}$ from the satellite to the sea surface in the nadir point direction. The altimeter transmits a short pulse of microwave radiation with known power toward the sea surface. The pulse interacts with the rough sea surface and part of the incident radiation reflects back to the altimeter. The techniques for radar determination of the time $t$ for the pulse to travel round trip between the satellite and the sea surface are described e.g. in Chelton and Ries ${ }^{[8]}$. The altimeter also measures the forms of the reflected pulses, named waveforms, from which other parameters of the ocean surface can be retrieved: significant wave height, maximum power and slope of the trailing edge. While the received peak power is connected to the altimeter normalized radar cross section $\sigma_{0}$, the slope of the waveform leading edge yields estimates of significant wave height (swh) and the trailing edge slope can provide an estimate of the radar antenna mispointing angle $(\xi)$. All these parameters can be converted to geophysical parameters by an algorithm called "retracking" that fits an analytical model to the measured waveforms. The Brown model ${ }^{[9]}$ is commonly used providing a good representation of the return echo over deep ocean surfaces that are assumed homogeneous. In many circumstances this has proved to be a very good model allowing for example precise retrievals of surface wind speed, swh and sea level anomalies (sla).

Of particular interest to this study are high-frequency variations of the altimeter normalized radar cross section $\sigma_{0}$, which using a geometric optics (specular) scattering model ${ }^{[5][6]}$ can be converted to mean square slope (mss), a useful measure of the roughness of a water surface with wind-driven waves. Thompson ${ }^{[10]}$ among others has pointed out that some attenuation of near-vertical backscatter can be due to diffraction effects caused by small scale (gravity-capillary) waves. The theory is that under a two-scale Gaussian approximation for the surface, the predominant backscatter will come from quasi-specular reflections due to long-scale slopes, but short-scale waves below the high-frequency (or cut-off) limit will diffract radiation in proportion to their height spectral density. Such an effect may skew the traditional filtered-surface (mean square slope) interpretation and also indicates potential for a second frequency dependency in altimeter backscatter modeling. The availability of both $\mathrm{Ku}$ and $\mathrm{C}$ radar frequency bands on the Jason altimeter series (Jason-2 for this study) allows isolation of the mean square slope contribution of the small scale waves with wavelengths roughly between $6.3 \mathrm{~cm}$ and $16.5 \mathrm{~cm}$. This gravity-capillary wavelength interval is highly affected by modulation due to the presence of short-period internal waves and internal solitary waves, hence the potential for internal wave detection with high sampling rate altimeters operating in dual-frequency bands.

\subsection{Internal Solitary Waves and sea surface roughness}

In the tropical ocean and along equatorial latitudes, the top $100 \mathrm{~m}$ to $300 \mathrm{~m}$ are several degrees warmer than water below, and this gives rise to a thermocline along which interfacial internal waves can propagate. Although the oscillations may typically have amplitudes of $100 \mathrm{~m}$ or more, internal waves (IWs) produce very small surface elevations, on the order of tens of $\mathrm{cm}$ or less. Still, the cellular currents that accompany them are of the same order as wave phase speeds, typically a few tens of centimeters per second to $3.5 \mathrm{~m} / \mathrm{s}$. The periodic spatial patterns of surface currents produce convergences and divergences strong enough to modulate short-length, surface gravity waves and capillary waves, resulting in a surface roughness signature characteristic of the underlying IW field. The sea surface roughness patterns produced by the internal wave-surface wave interaction are responsible for making them visible to imaging sensors such as synthetic aperture radars (SARs) and moderate-resolution optical sensors such as MODIS (the Moderate Resolution Imaging Spectroradiometer from NASA).

Hydrodynamic modulation theory describes the evolution of small-amplitude surface waves in a slowly varying current, and it is derived from the action balance equation (see, e.g., Apel ${ }^{[11]}$ ), which accounts for the conservation of wave energy where the wavenumber and frequency of the wave field vary in space and time ${ }^{[12]}$. A common assumption has been that the variable surface current due to the orbital velocity of IWs leads to only small deviations from the equilibrium wave spectrum so that the action balance equation can be solved retaining only first-order terms (see e.g. 
Alpers ${ }^{[3]}$ ). Consequently, for oblique radar looking radars, Bragg scattering can explain the mechanism for radar cross section modulation in the IW field, and account for the spectacular surface manifestations of IWs in satellite radar images.

The modulation effect of IWs on sea surface roughness can be readily demonstrated by measurements of wind wave slope variances associated with short-period ISWs, as accomplished in the pioneer work of Hughes and Grant ${ }^{[4]}$. These authors were amongst the first to provide experimental and theoretical support for the idea that surface slope statistics are closely related to the internal wave currents. More recently, Kudryavtsev ${ }^{[13]}$ developed a transfer function that relates the sun glitter brightness contrast to the mean square slopes contrasts. Their method provides new opportunities for quantitative investigations of surface signatures of ocean IWs in MODIS images, possibly with synergy between altimeter estimates of mean square slope and sun glitter imagery.

\section{METHODOLOGY.}

A useful measure of the roughness of a water surface with wind-driven waves is the mean square surface slope (mss), defined as

$$
\left\langle s^{2}\right\rangle=\int_{0}^{\infty} S(\kappa) \kappa d \kappa
$$

where $S(\kappa)$ is the omnidirectional, one-sided wave number slope spectrum and $\kappa$ is the wave number ${ }^{[14]}$. In this study we consider only the mean square slope contribution of waves within a specific wave number range by integrating Eq. (1) over the limits $\kappa_{1}-\kappa_{2}$, where $\kappa_{1}$ and $\kappa_{2}$ bracket a range of wave numbers contributing strongly to IW roughness modulation. The result of the definite integral in Eq. (1) is designed as $\left\langle s^{2}\right\rangle_{\kappa 1}^{\kappa 2}$. Here, the wave numbers $\kappa_{l}$ and $\kappa_{2}$, are chosen to correspond to the upper wave number limits that satisfy the quasispecular scattering condition of the geometrical optics model for the two radar wavelengths used on the Jason-2 altimeters ( $\mathrm{Ku}$ and C-bands). Thus defined $\left\langle s^{2}\right\rangle_{\kappa 1}^{\kappa 2}$ can then be compared with the difference in mean square slope estimates derived from Ku-band and C-band backscatter.

The mean square slope of waves in the wave number range that satisfies specular scattering conditions is inversely related to the normalized backscatter ${ }^{[5][6]}$. Assuming the surface wavefield as an isotropic Gaussian surface, as Cox and Munk ${ }^{[15]}$ did in their pioneer work of slope statistics, it is possible to apply the geometrical optics (GO) form of the integrated microwave backscatter cross section according to the expression

$$
\sigma_{0}^{G O}=\left(\rho_{g} \sec ^{4} \theta /\left\langle s_{g}^{2}\right\rangle\right) e^{\left(-\tan ^{2} \theta /\left\langle s_{g}^{2}\right\rangle\right)}
$$

where $\rho_{g}$ is an effective reflectivity, $\left\langle s_{g}{ }^{2}\right\rangle$ is an effective mean square slope estimate and $\theta$ is the pulse illumination incidence angle. Although Eq. 2 neglects anisotropy of the ocean surface with respect to slope ${ }^{[16]}$ the isotropic form of the integrated cross section leads at most to an error of a few percent in the estimation of mean square slope ${ }^{[6]}$. For satellite altimeter observations (near-nadir, i.e. $\theta \sim 0$ ), Eq. 2 reduces to give mean square slope as

$$
\left\langle s_{n}^{2}\right\rangle=\frac{\rho_{n}^{\prime}}{\sigma_{0}}
$$

where the subscript $n$ is used to indicate nadir and $\rho_{n}^{\prime}$ is understood to differ from a pure Fresnel reflectivity coefficient in that it may include diffraction effects ${ }^{[7]}$. 
The effective mean square slope variable $\left\langle s_{n}{ }^{2}\right\rangle$ given by (3) can be evaluated from either $\sigma_{0}{ }^{\mathrm{C}}$ or $\sigma_{0}{ }^{\mathrm{ku}}$, but differs from the total mean square slope as measured by optical methods (e.g., Cox and Munk ${ }^{[15]}$ ) in that it represents an integration of the wave number slope spectrum (1) only up to a cutoff wavenumber corresponding to C-band and Ku-band radar wavelengths, respectively. Hence, these mean square slopes include contributions from all wave facets with dimensions greater than a cutoff wavelength $\lambda_{\text {cutoff }} \sim 3 \lambda_{i}$ where $\lambda_{i}$ is the radar wavelength ${ }^{[5][17]}$. For the Jason- $2 \mathrm{Ku}$-band $\left(\lambda_{i}=2.1 \mathrm{~cm}\right)$ and C-band $\left(\lambda_{i}=5.5 \mathrm{~cm}\right)$ channels, these cutoff wavelengths are roughly $6.3 \mathrm{~cm}$ and $16.5 \mathrm{~cm}$, respectively, which allows isolation of the mean square slope contribution of the small-scale waves between $6.3 \mathrm{~cm}$ and $16.5 \mathrm{~cm}$ by differencing the estimates from the two frequency bands. Since footprint sizes for both Ku-band and C-band altimeter frequencies are the same for the Jason- ${ }^{[7]}$, meaning that the areas sampled by the two radar pulses are identical, the difference mean square slope eliminates backscatter contributions from the longer waves. This methodology, proposed in Chapron ${ }^{[18]}$ and applied in Frew ${ }^{[7]}$ to estimate global air-sea gas transfer velocity, is here introduced as a proxy to detect short-period internal waves. It relies on the fact that short-period internal waves strongly modulate the surface wave spectrum in this shortwavelength range for various reasons. Along with the above mentioned interaction between varying IW currents and wind-generated gravity-capillary waves (i.e. weak hydrodynamic modulation theory), one must also assume other modulations such as those produced by variations of surface film concentration ${ }^{[19]}$, IW relative wind speed variability ${ }^{[20]}$ and specular reflection from breaking surface waves and Bragg scattering from small-scale surface waves generated by wave breaking ${ }^{[21]}$.

Radar altimeter signals are attenuated by raindrops from both absorption and scattering. The effects of rain contamination are often apparent from erratic variation of $\sigma_{0}$ as well as significant wave height ${ }^{[8]}$. Since rain attenuation is an order of magnitude larger at $\mathrm{Ku}$-band than at $\mathrm{C}$-band, rain-contaminated observations from the Jason-2 dualfrequency altimeter can usually be identified as an abrupt decrease in Ku-band $\sigma_{0}$ relative to C-band $\sigma_{0}$. However, in this study we cannot rely on this criterion on its own to discard rain affected measurements, as those differenced dualfrequency $\sigma_{0}$ fluctuations may also be due to IW surface manifestations. At present, our method to deal with rain affected measurements consists of a threshold in the integrated columnar liquid water content $L_{z}$, here chosen as $0.01 \mathrm{~g} . \mathrm{cm}^{-2}$. All radiometer measurements not satisfying $L_{z}<0.01 \mathrm{~g} . \mathrm{cm}^{-2}$ are discarded as suspicious of rain. This paper is based on synergy observations between altimeter and moderate resolution images affected by sunglint with clear signatures on internal solitary waves. The selected images (mostly MODIS in the visible wavebands) are hardly affected by clouds, therefore we believe that the threshold liquid water content $L_{z}$ and visual inspection are suffice to deal with unwanted rain events.

\section{RESULTS}

The results presented in this paper correspond to processing and analysis of the Jason-2 Sensor Geophysical Data Records (SGDR), in high resolution (i.e. 20-Hz data), for cycles $0-291$, spanning the period 4 July, 2008 to 5 June, 2016. The SGDRs were obtained from ftp.nodc.noaa.gov by ftp. Suitable passes covering regions were powerful internal waves have been reported in the literature were selected ${ }^{[22]}$. The following altimeter derived geophysical parameters were chosen for analysis: the ku-band radargrams (or waveforms) corrected for the on-board power tracker loop AGC (Automatic Gain Control); the normalized radar backscatter $\sigma_{0}$ in C-band and Ku-band as retrieved from the retracking algorithms Maximum Likelihood Estimator (MLE) MLE4 and MLE3; the mean square slopes for the nominal wavenumber range $40-100$ rad. $\mathrm{m}^{-1}$ derived from those backscatter measurements; the along-track off-nadir angle provided by the MLE4 retracker; and the significant wave height.

Precise estimates of geophysical parameters are obtained thanks to an algorithm called "retracking" that fits an analytical model to the measured waveforms. The MLE3 algorithm estimates three parameters (range, significant wave height, and power) whereas the MLE4 estimates four parameters (the three previous ones and the slope of the waveform trailing edge) and was introduced to deal with larger antenna mispointing angles that arose from abnormal behavior in the star tracker system of Jason-1 after launch. It is important to note that the MLE4 algorithm was adopted for the Jason-2 
nominal waveform retracking despite the good pointing performance of the Jason-2 satellite. Thibaut ${ }^{[23]}$ provided clear arguments to the benefit of MLE4 algorithm for range and significant wave height estimates especially when the return echoes do not conform to the Brown model (admittedly the case of interest in this paper). However, Thibaut ${ }^{[23]}$ acknowledges a degraded estimation of the sigma naught coefficient from MLE4 in relation to MLE3, and notes that the MLE4 sigma naught may be correlated with the antenna mispointing angle. We think that this notable correlation might not actually be unphysical and show that it sometimes coincides with internal wave signatures detected in the MODIS imaging sensor.

In the following we present selected case studies for three different regions with powerful occurrences of internal solitary waves (ISWs): Mascarene Ridge of the Indian Ocean; tropical deep Atlantic Ocean off the Amazon river mouth; and the Andaman Sea. When evaluating equation (3) for retrieving mean square slopes for the two radar bands (Ku and $\mathrm{C}$ ) we used $\rho_{n}{ }^{C}=0.617$ and $\rho_{\mathrm{n}}{ }^{\mathrm{ku}}=0.427$ according to the optimized parameters reported in Frew et al. ${ }^{[7]}$ (see their Table 1).

\subsection{Mascarene Ridge of the Indian Ocean}

On December 11, 2015, Jason-2 overpassed the Mascarene Ridge of the Indian Ocean (Pass 029; Cycle 274) at 02:51

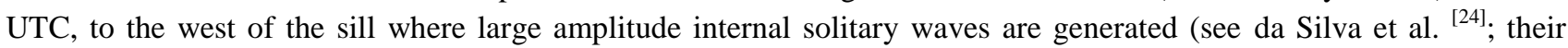
Figure 4). A MODIS Aqua image acquired at 09:35 UTC over the same region reveals a large ISW train propagating to the west, whose wave fronts extend for at least $200 \mathrm{~km}$ approximately along the direction of the altimeter track (see red line in Fig. 1a). In Fig. 1a the position of the wave features were corrected for the time gap between the two satellite observations, since ISWs propagate with velocities as high as $3.0 \mathrm{~m} / \mathrm{s}$ in that region (see da Silva et al., 2011). Assuming a phase speed of $3.0 \mathrm{~m} / \mathrm{s}$ and a wave propagation direction of $300{ }^{\circ} \mathrm{TN}$, during the $6 \mathrm{~h} 44 \mathrm{~min}$ between the Jason-2 overpass and the MODIS overpass the waves (and their surface manifestations) moved $0.5657^{\circ}$ in longitude to the West and $0.3266^{\circ}$ to the North. Hence, we subtracted those distances to the MODIS image in Fig. 1a in order to obtain the wave-train location by the time of Jason-2 overpass.

Fig. 1b shows geophysical parameters as described above for Jason-2 Pass 029 Cycle 274. Of particular interest is the backscatter perturbation (Ku-band) at approximately $-11.2{ }^{\circ} \mathrm{N}$ that coincides with a signature in the radargram and a significant decrease in the off-nadir angle. Note that the mss around $-11.2^{\circ} \mathrm{N}$ derived from the ku-band backscatter (dark signal) follows the backscatter perturbation (as expected) and varies from 0.015 to 0.025 in about $10 \mathrm{~km}$ distance, while the C-band retrieved mss is much less perturbed. These mss modulations coincide with the positions of the ISW features depicted in Fig. 1a. We also note that, in this case, there are no signatures of the ISW in the SHW field provided at the bottom of Fig. 1b. 

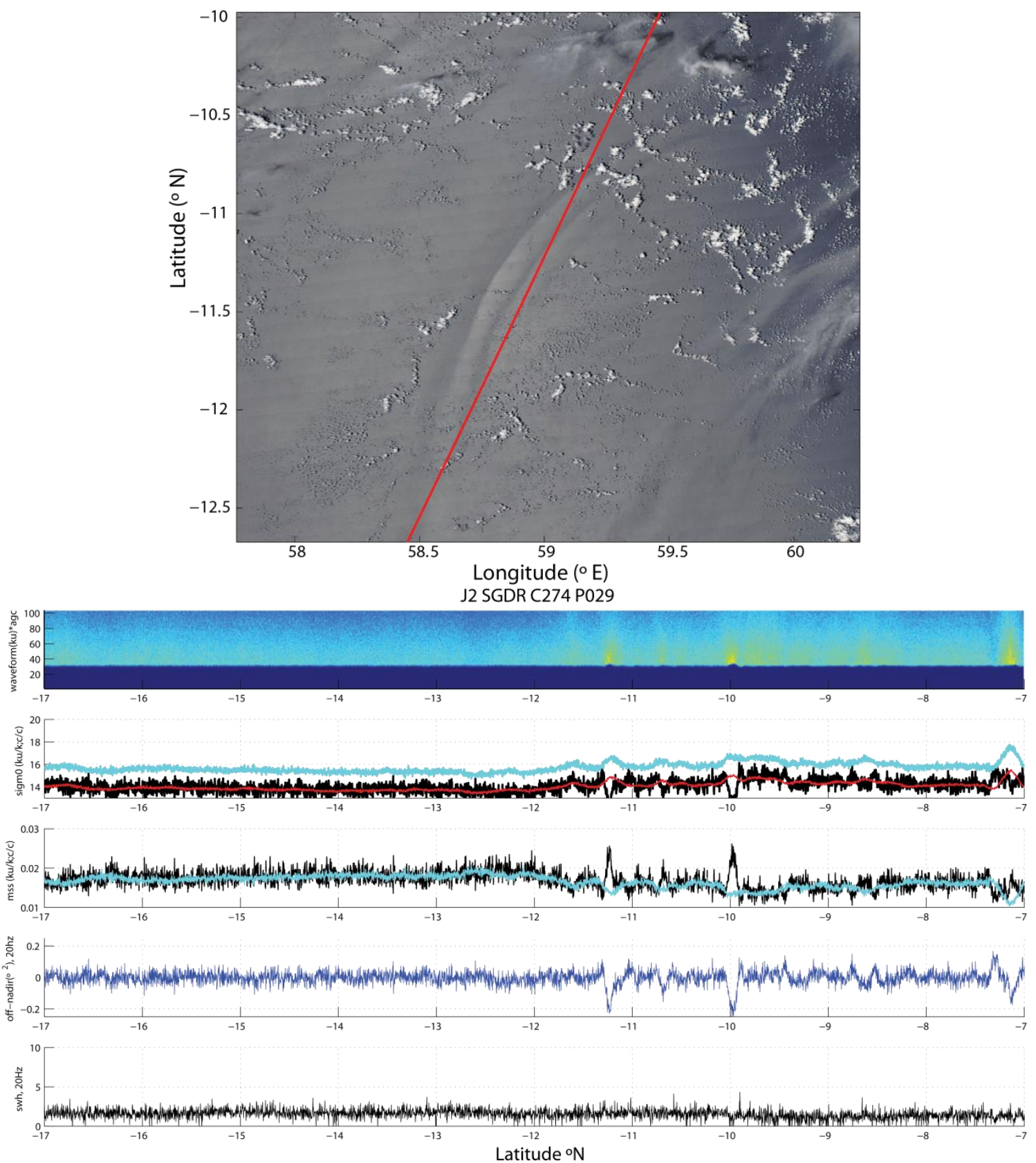

Figure 1. Modis Aqua image processed in RGB (quasi- true color) (top) showing a train of ISWs and the Jason-2 overpass (029) corresponding to cycle 274 on 11 December, 2015. Retrieved geophysical parameters (from top to bottom): waveforms for ku-band; along-track radar backscatter sigma0 (ku-band in black using MLE4; ku-band in red using MLE3; c-band in light blue; mean square slope derived from sigma0 (same colors); off-nadir angle (dark blue); significant wave height derived using MLE4.

\subsection{Amazon tropical Atlantic Ocean}

On September 22, 2012, Jason-2 overpassed the equatorial Atlantic Ocean (Pass 176; Cycle 155) at 21:30 UTC, to the North of the Amazon shelf break where large amplitude internal solitary waves are generated (see Magalhaes et al. ${ }^{[25]}$; their Figure 3a). A MODIS Aqua image acquired at 16:10 UTC over the same region reveals several large ISW trains 
propagating to the northeast, whose wave fronts extend for at least $300 \mathrm{~km}$ approximately across the direction of the altimeter track (see red line in Fig. 2a).
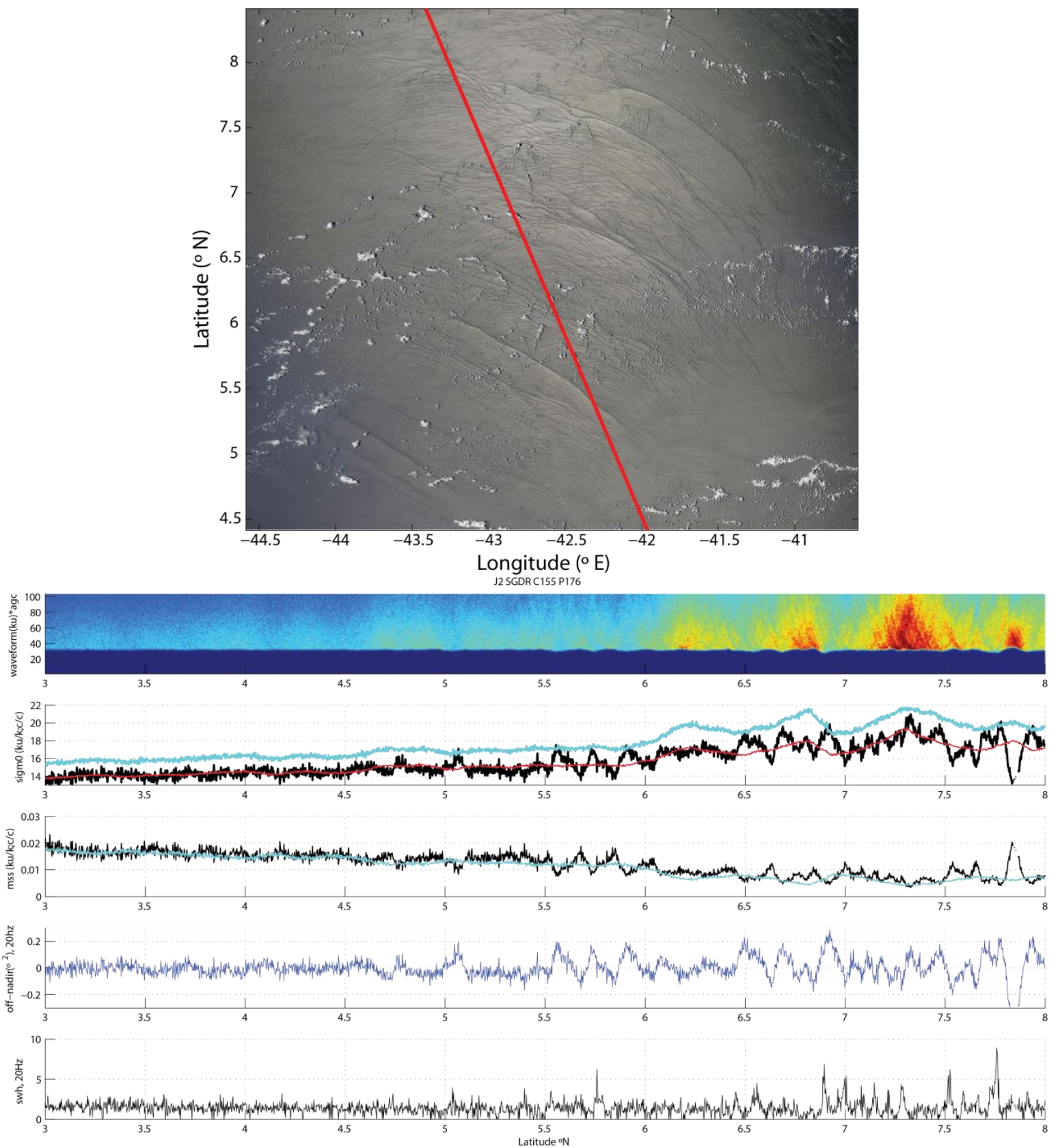

Fig. 2. Modis Aqua image processed in RGB (quasi- true color) (top) showing a train of ISWs and the Jason-2 overpass (176) corresponding to cycle 155 on 22 September, 2012. Retrieved geophysical parameters (from top to bottom): waveforms for ku-band; along-track radar backscatter sigma0 (ku-band in black using MLE4; ku-band in red using MLE3; c-band in light blue; mean square slope derived from sigma0 (same colors); off-nadir angle (dark blue); significant wave height derived using MLE4.

As in the previous study case, the positions of the wave features in Fig. 2a were corrected for the time gap between the two satellite observations, since ISWs propagate with velocities as high as $3.4 \mathrm{~m} / \mathrm{s}$ off the Amazon shelf (see Magalhaes 
et al. ${ }^{[25]}$ ). Assuming a phase speed of $3.4 \mathrm{~m} / \mathrm{s}$ and a wave propagation direction of $045{ }^{\circ} \mathrm{TN}$, during the $5 \mathrm{~h} 20 \mathrm{~min}$ between MODIS overpass and the Jason-2 overpass, the waves (and their surface manifestations) moved $0.413^{\circ}$ in longitude to the East and $0.413^{\circ}$ to the North. Hence, we add those distances to the MODIS image in Fig. 2a in order to obtain the wave location by the time of Jason-2 overpass.

Fig. 2b shows geophysical parameters for Jason-2 Pass 176 Cycle 155. Of particular interest are backscatter perturbations (Ku-band) from $5{ }^{\circ} \mathrm{N}$ to $8^{\circ} \mathrm{N}$ that coincide with $\mathrm{V}$-shape signatures in the radargram and a significant modulation of the off-nadir angle. Note that the mss in between $5-8{ }^{\circ} \mathrm{N}$ derived from the ku-band backscatter (dark signal) follows the backscatter perturbations (as expected) and varies with amplitudes as much as 0.01 in turn of the much smoother mss derived from the c-band backscatter. These oscilations occur for more than $300 \mathrm{~km}$ along-track. Here too, the mss modulations coincide with the positions of the ISW features depicted in Fig. 2a. Note that in this case some of the ISWs detected in the sigma0 and "off-nadir-angle" fields also appear as "spikes" in the SWH along-track record (bottom graphic in Fig. 2b).

\subsection{Andaman Sea}

On March 20, 2015, Jason-2 overpassed the northern Andaman Sea (Pass 090; Cycle 247) at 18:40 UTC, where large amplitude internal solitary waves were observed propagating towards the Andaman Islands. A MODIS Terra image acquired at 04:25 UTC over the same region reveals several large ISW trains propagating to the west-southwest, whose wave fronts extend for at least $100 \mathrm{~km}$ approximately along the direction of the altimeter track (see red line in Fig. 3a). In this case the time difference between the overpasses of the two satellites exceeds a semi-diurnal tidal cycle (12.42 hours) in approximately two hours. The tides in the Andaman Sea hold a strong semi-diurnal component, being capable to generate large amplitude ISWs in successive tidal cycles at approximately the same locations every $12 \mathrm{~h} 25 \mathrm{~min}$. This means that the ISW structure would repeat itself (similar wave trains) every $12 \mathrm{~h} 25 \mathrm{~min}$, holding the same structure as long as the internal stratification does not change too much. Consequently, images like those in Fig. 3a should be very similar every tidal cycle, and positions of ISW trains should be almost the same. The time difference between the two satellite observations (altimeter and MODIS) is $14 \mathrm{~h} 20 \mathrm{~min}$, which means one tidal cycle plus 2 hours approximately. Hence, we choose not to correct the wave trains in Fig. 3a to their expected positions at the time of the altimeter overpass. Expected differences in ISW train positions should not exceed $10 \mathrm{~km}$ between the altimeter observation and their effective location at that time.

As before, Fig. 3b shows geophysical parameters for Jason-2 Pass 090 Cycle 247. Here our focus is on backscatter perturbations (Ku-band) from $11.5^{\circ} \mathrm{N}$ to $12.7^{\circ} \mathrm{N}$ that coincide with some kind of sigma0 bloom signatures in the radargram and a significant modulation of the off-nadir angle. The mss in between $11.5^{\circ} \mathrm{N}$ to $12.7^{\circ} \mathrm{N}$ derived from the ku-band backscatter (dark signal) follows the backscatter perturbations (as expected) and varies with amplitudes as much as 0.005 in turn of the much smoother mss derived from the c-band backscatter. These oscilations occur for more than $100 \mathrm{~km}$ along-track. As in previous examples, the mss modulations coincide with the positions of the ISW features depicted in the MODIS sensor shown in Fig. 3a. In this particular case we note some missing data near $11.9^{\circ} \mathrm{N}$, probably due to the failure of the MLE4 retracking algorithm to deal with the measured waveforms' power. In this case too, note the appearance of "spiky-features" along-track in the SWH field, which are obviously unphysical features since it is unlikely to observe a departure from SWH from an average of $2 \mathrm{~m}$ to values of 5-10 $\mathrm{m}$ in a short distance as suggested from the graphic in Fig. 3b (see bottom graphic). Although reports of "ripplings in calm weather" exist in the literature describing ISWs in the Andaman Sea, which refer to meter-scale surface waves of considerable amplitude and wave breaking associated to ISWs, wave heights are unlikely to reach values as much as $8 \mathrm{~m}$ as Fig. $3 \mathrm{~b}$ suggests. 


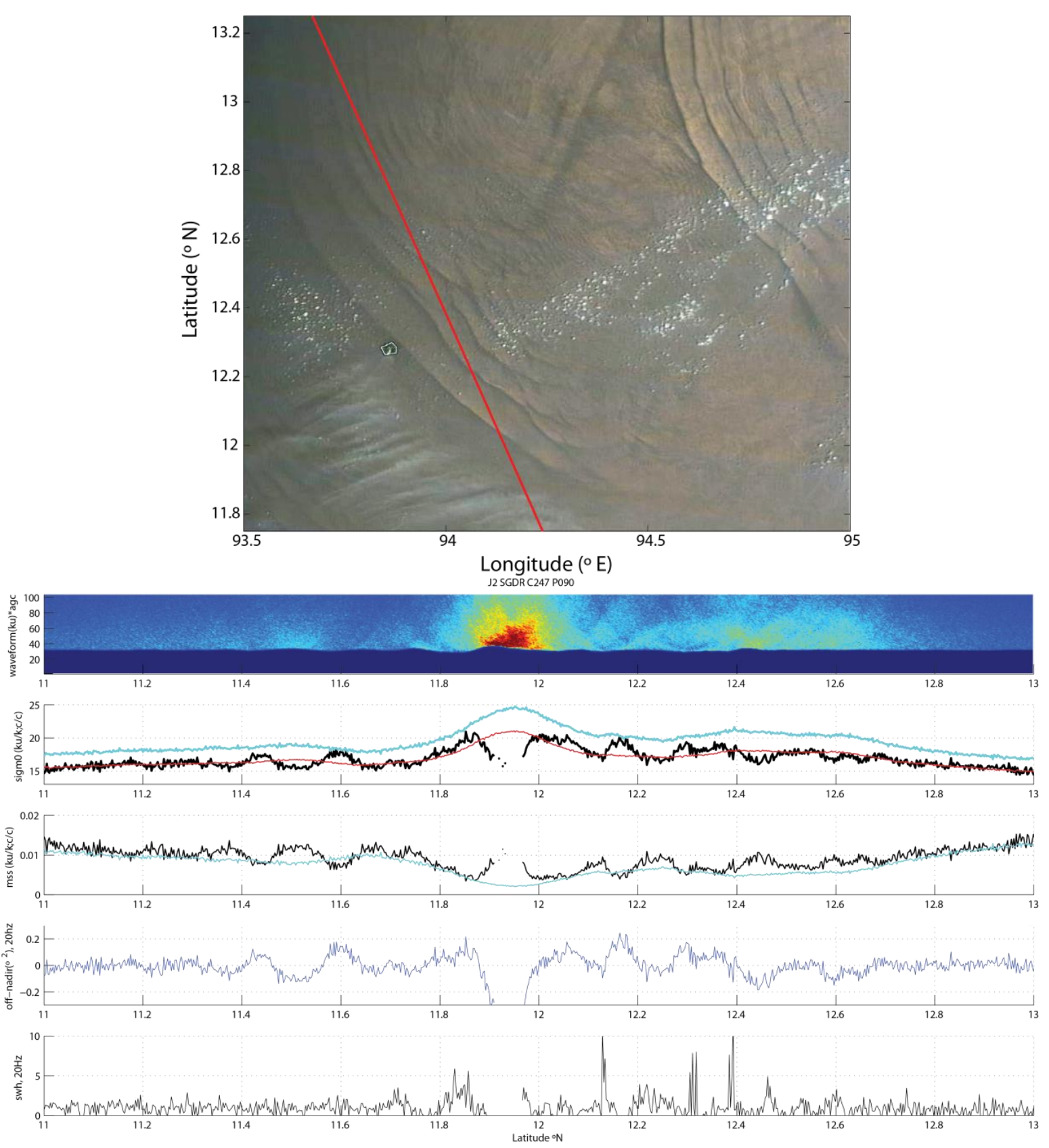

Fig. 3. Modis Terra image processed in RGB (quasi- true color) (top) showing several trains of ISWs and the Jason-2 overpass (090) corresponding to cycle 247 on 20 March, 2015. Retrieved geophysical parameters (from top to bottom): waveforms for ku-band; along-track radar backscatter sigma0 (ku-band in black using MLE4; ku-band in red using MLE3; c-band in light blue; mean square slope derived from sigma0 (same colors); off-nadir angle (dark blue); significant wave height derived using MLE4.

The examples provided in this Section correspond to a selection of synergy cases (between an imaging sensor such as MODIS and along-track high rate altimeter data) aiming to illustrate the existence of signatures of short-period internal waves in altimeter data. The character of these signatures is not yet fully understood and in this paper we merely want to demonstrate their existence. MODIS cloud free data helps to discard cases possibly contaminated by rain. In addition, in 
all the above cases, the threshold in the integrated columnar liquid water content satisfying $L_{z}<0.01 \mathrm{~g} . \mathrm{cm}^{-2}$ has been respected.

\section{DISCUSSION}

Considerations about the observed signal presumably generated by the surface roughness manifestations of short-period internal waves is two-fold: on one hand there is the theoretical prediction that differential scattering from the dual-band Jason-2 altimeter should be effective to isolate the contribution of small-scale surface waves to mean square slope variations due to ISWs; on the other hand current retracking algorithms, based on the Brown model, may originate unphysical artifacts in response to inhomogeneities in backscatter strength ${ }^{[26]}$.

At stake here are some systematic response and differences between MLE3 and MLE4 retracking algorithms. Thibaut et al. ${ }^{[23]}$ reported the benefits of using MLE4 during sigma0 bloom events such as those reported here in the presence of internal waves. Admittedly, MLE4 provides better retracked parameters when waveform trailing edges are affected by spurious signatures that might be mixed up with true satellite mispointing. Dibarboure et al. ${ }^{[26]}$ points out that, in the case of MLE3, the fitted waveforms underestimate the amplitude and are unable to reproduce artifacts in the measured waveforms trailing edges (see their Figure 9). These authors demonstrate that, while for a Brownian waveform MLE3 and MLE4 are very consistent and the retracked parameters are the same, when the measured waveform is significantly distorted by a bloom event, MLE3 and MLE4 exhibit major differences. Because MLE4 estimates the slope of the trailing edge, it is able to better fit disturbed waveforms. We systematically observe strongly reduced sigma0 modulations of MLE3 compared to MLE4 in our internal wave records.

With MLE4, the improved Brown model provides a new capability to absorb the waveform distortion as "off-nadir angle" and sigma0 (note that the true pointing of Jason-2 is very good, so the retracked off-nadir angle is only apparent). Here we suggest that the "off-nadir angle" may be used as a parameter to detect short-period internal waves, and that the sigma0 coefficient estimations from MLE4 are improved in relation to MLE3 (contrary to what has been suggested by Thibaut et al. ${ }^{[23]}$ ).

Figure 4 shows illustrations of typical waveform distortions due to sea surface roughness variations along short-period internal waves. The records correspond to the study case presented above and dated March 20, 2015, when Jason-2 overpassed the Andaman Sea at 18:40 UTC during Cycle 247 (Pass 090). In part a) of Fig. 4 we present the radargram of a sector unperturbed by the presence of ISWs (latitude ranging from 11.0 to $11.3^{\circ} \mathrm{N}$ ) whose waveforms are Brownian in nature. Part b) depicts a sector where some perturbation is observed (latitude ranging from 11.78 to $11.88{ }^{\circ} \mathrm{N}$ ), presumably due to the leading front of a large ISW where strong current convergence at the sea surface leads to roughness enhancement and departure from Brownian waveform shape. In part c) of Fig. 4 a strong backscatter perturbation admittedly coincides with the presence of a large sigma0 bloom (latitude ranging from 11.88 to $11.98{ }^{\circ} \mathrm{N}$ ) associated to a slick band in the rear slopes of the same ISW. Note that the backscatter power in the sigma0 bloom sector is three-fold higher than the observed values in the sector unperturbed by the ISWs. Furthermore, the trailing edge of the averaged altimeter waveforms (thick light blue curve) is much steeper than the unperturbed averaged trailing edge waveform (thick red curve in part a). Note that in both sectors depicted as "perturbed" the averaged waveform shapes (thick green and light blue curves) are significantly different from the Brownian shape (thick red curve). 

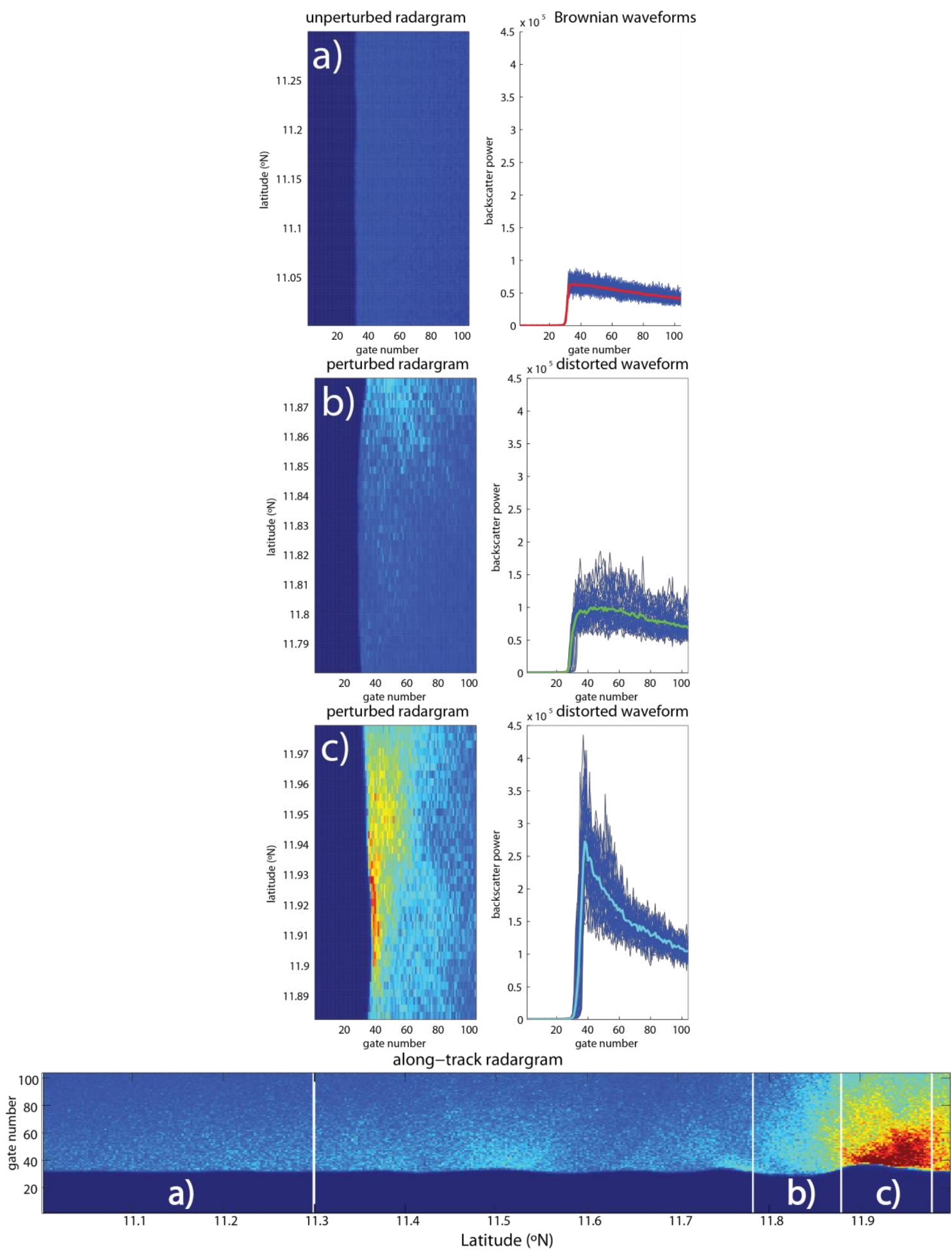

Fig. 4. From top to bottom: a) unperturbed waveforms in the absence of short-period internal waves or slicks; b) leading front of an ISW (average waveform power is given by green curve); c) rear slope of an ISW characterized by divergence and existence of a sigma0 bloom (average waveform power is given by light blue curve); d) corresponding along-track radargram showing transition from unperturbed wave field to disturbed non-brownian waveforms. Note that power backscatter is in the same scale for all cases (see text for details). 
All in all, we observe evidence of ISWs in the following altimeter geophysical parameters: 1) the apparent "off-nadirangle"; 2) sigma0 as retrieved from MLE4; 3) SWH as retrieved from MLE4; and 4) the differenced mean square slope calculated from the dual-band Jason-class altimeters. In addition, analysis of the along-track radargram reveals paraboliclike features, sometimes denominated v-shaped features, which are associated to short-scale sigma0 bloom events that have been modelled as sigma0 Dirac positive anomalies (i.e. a straight line or band of singular corrupted pixels associated to a slick band with the same width as the radar altimeter resolution, which is approximately $300 \mathrm{~m}$ ) by Dibarboure et al. ${ }^{[26]}$ and Tournadre et al. ${ }^{[27]}$. These authors further note that a similar shape anomaly is expected in the radargram if the sigma0 anomaly is negative (i.e. less power returned in a single $300 \mathrm{~m}$ cell). We have noted both types of parabolic-like sigma0 anomalies in the many radargrams we have analyzed, which we think might be associated to roughness modulations induced by ISWs at the sea surface.

\section{SUMMARY AND CONCLUSIONS}

In this paper we developed efforts for detection and recognition of short-period internal waves, also many times described in the literature as ISWs, in high-rate along-track records of the Jason-2 altimeter. Geophysical parameters obtained from available SGDRs were processed and analyzed for regions of the global ocean where the existence of high-amplitude ISWs is known, namely: the Mascarene Ridge of the Indian Ocean, the tropical Atlantic Ocean off the Amazon shelf and the Andaman Sea. Evidence of modulation of several geophysical parameters was presented in this paper, namely: the "off-nadir-angle" available from the MLE4 retracking algorithm; sigma0 as retrieved from MLE4; significant wave height (SWH); and the differenced mean square slope calculated from the dual-band Jason-2 altimeter.

The ISW signatures are sometimes recognized as parabolic-like shape sigma0 anomalies in the along-track radargram. These anomalies are mostly recognized as sigma0 positive anomalies which are related to short-event sigma0 blooms that have been reported in the literature ${ }^{[26]}{ }^{[27]}$. On some occasions however, sigma0 negative anomalies with paraboliclike shape have also been observed back-to-back with the positive anomalies. We suggest that these consecutive negative/positive anomalies are associated to enhanced and decreased surface roughness produced by ISWs. This is consistent with some of our records of differenced mean square slope calculated from the dual-band Jason-2 altimeter.

For the present application, detection and recognition of short-period internal waves, the improved MLE4 Brown model provides the capability to absorb the waveform distortion as "off-nadir angle" and sigma0 (note that the true pointing of Jason-2 is very good, so the retracked off-nadir angle is only apparent). With MLE4 the slope of the trailing-edge parameter and the sigma0 estimate are absorbing the bulk of the backscattering event when the outer rings of the waveform footprint are affected. This paper shows that oceanography users interested in short-period internal wave signals may find useful information in $20-\mathrm{Hz}$ rate Jason- 2 current altimeter products. Development of better editing and postprocessing algorithms on the $20-\mathrm{Hz}$ rate of current products is needed if we want to develop quantitative information about short-period internal waves detected in current and new generation altimeters.

\section{ACKNOWLEDGEMENTS}

The first author would like to thank Alexis Mouche for stimulating discussions about the possibility of detection of internal solitary waves with new generation altimeters.

\section{REFERENCES}

[1] Ray, R. D., Mitchum, G. T. , "Surface manifestation of internal tides generated near Hawaii," Geophys. Res. Let. 23(16), 2101-2104 (1996). 
[2] Hughes, B. A., "The effect of internal waves on surface wind waves, 2. Theoretical analysis," J. Geophys. Res. 83(1), 455-465 (1978).

[3] Alpers, W., "Theory of radar imaging of internal waves," Nature 314, 245-247 (1985).

[4] Hughes, B. A., Grant, H. L., "The Effect of Internal Waves on Surface Wind Waves, 1. Experimental Measurements," J. Geophys. Res. 83, 443-454 (1978).

[5] Brown, G. S., [Quasi-specular scattering from the air-sea interface, in Surface Waves and Fluxes], Vol. 2, Springer, New York, 1-40 (1990).

[6] Jackson, F. C., Walton, W. T., Hines, D. E., Walter, B. A., Peng, C. Y., "Sea surface mean square slope from Ku-band backscatter data," J. Geophys. Res., 97(C7), 11411-11427 (1992).

[7] Frew, N. M., Glover, D. M., Bock, E. J., McCue, S. J., "A new approach to estimation of global air-sea gas transfer velocity fields using dual-frequency altimeter backscatter," J. Geophys. Res. 112, 11003 (2007)

[8] Chelton, D.B., Ries, J.C., Haines, Bruce J., Fu, Lee-Lueng and Callahan, Philip S., [Satellite Altimetry, Chapter 1], Jet Propulsion Laboratory, Pasadena California, 1-183 (2000).

[9] Brown, G. S., "The average impulse response of a rough surface and its application," IEEE Trans. Antennas Propag. 25, 67-74 (1977).

[10] Thompson, D. R., "Calculation of radar backscatter modulations from internal waves," J. Geophys. Res. 93, 12371-12380 (1988).

[11] Apel, J. R., [Principles of Ocean Physics], Academic Press, San Diego, CA., 1-631 (1987)

[12] Bretherton, F. P., Garrett, C. J. R., "Wave trains in inhomogeneous moving media," Proc. R. Soc. Lond. A, 301, 539 (1968).

[13] Kudryavtsev, V., Myasoedov, A., Chapron, B., Johannessen, J. A., Collard, F., "Joint sun-glitter and radar imagery of surface slicks," Remote Sensing of Environment 120, 123-132 (2012).

[14] Hara, T., Bock, E. J., Edson, J. B., McGillis, W. R., "Observations of short wind waves in coastal waters," J. Phys. Oceanogr. 28, 1425-1438 (1998).

[15] Cox, C., Munk, W., "Statistics of the sea surface derived from sun glitter," J. Mar. Res. 13(2), 198- 227 (1954).

[16] Liu, Y., Su, M.-Y., Yan, X.-H., Liu, W. T., "The mean square slope of ocean surface waves and its effects on radar backscatter," J. Atmos. Oceanic. Technol. 17, 1092-1105 (2000).

[17] Elfouhaily, Chapron, T., B., Katsaros, K., Vandemark, D., "A unified directional spectrum for long and short wind-driven waves," J. Geophys. Res. 102, 15781-15796 (1997).

[18] Chapron, B., Katsaros, K., Elfouhaily, T., Vandemark D., "A note on relationships between sea surface roughness and altimeter backscatter," Air-Water Gas Transfer, 869 - 878 (1995).

[19] da Silva, J.C.B., Ermakov, S.A., Robinson, I.S., Jeans, D.R.G., Kijashko, S.V., "Role of surface films in ERS SAR signatures of internal waves on the shelf - 1. Short-period internal waves," J. Geophys. Res. 103, 8009-8031 (1998).

[20] Ermakov, S.A., da Silva, J.C.B., Robinson, I.S., "Role of surface films in ERS SAR signatures of internal waves on the shelf - 2. Internal tidal waves," J. Geophys. Res. 103, 8032-8043 (1998).

[21] Kudryavtsev, V., Akimov, D., Johannessen, J.A., Chapron, B., "On radar imaging of current features: 1. Model and comparison with observations," J. Geophys. Res. 110, 07016 (2005).

[22] Jackson, C.R., da Silva, J.C.B., Jeans, G., "The generation of nonlinear internal waves," Oceanography 25(2), 108-123 (2012).

[23] Thibaut, P., Poisson, J. C., Bronner, E., Picot, N., "Relative performance of the MLE3 and MLE4 retracking algorithms on Jason-2 altimeter waveforms," Mar. Geod. 33, 317-335 (2010).

[24] da Silva, J.C.B., New, A.L., Magalhaes, J.M., "On the structure and propagation of internal solitary waves generated at the Mascarene Plateau in the Indian Ocean," Deep-Sea Research I 58, 229-240 (2011). 
[25] Magalhaes, J.M., da Silva, J. C. B., Buijsman, M. C. , Garcia, C. A. E., "Effect of the North Equatorial Counter Current on the generation and propagation of internal solitary waves off the Amazon shelf (SAR observations)," Ocean Sci. 12, 243-255 (2016).

[26] Dibarboure, G., Boy, F., Desjonqueres, J.D. , Labroue, S., Lasne, Y., Picot, N., Poisson, J.C., Thibaut P., "Investigating Short-Wavelength Correlated Errors on Low-Resolution Mode Altimetry," Journal of Atmospheric and Oceanic Technology 31, 1337-1362 (2014).

[27] Tournadre, J., Chapron, B., Reul, N., Vandemark, D. C., "A satellite altimeter model for ocean slick detection," J. Geophys. Res. 111, 04004 (2006). 